\title{
REGULAR INJECTIVITY AND EXPONENTIABILITY IN THE SLICE CATEGORIES OF ACTIONS OF POMONOIDS ON POSETS
}

\author{
Farideh Farsad and Ali Madanshekaf
}

\begin{abstract}
For a pomonoid $S$, let us denote Pos- $S$ the category of $S$-posets and $S$-poset maps. In this paper, we consider the slice category Pos- $S / B$ for an $S$-poset $B$, and study some categorical ingredients. We first show that there is no non-trivial injective object in Pos- $S / B$. Then we investigate injective objects with respect to the class of regular monomorphisms in this category and show that Pos- $S / B$ has enough regular injective objects. We also prove that regular injective objects are retracts of exponentiable objects in this category. One of the main aims of the paper is to draw attention to characterizing injectivity in the category Pos- $S / B$ under a particular case where $B$ has trivial action. Among other things, we also prove that the necessary condition for a map (an object) here to be regular injective is being convex and present an example to show that the converse is not true, in general.
\end{abstract}

\section{Introduction and preliminaries}

A slice category is a construction in category theory which provides another way of looking at morphisms: instead of simply relating objects of a category to one another, morphisms become objects in their own right. This notion was introduced in 1963 by F. W. Lawvere, although the technique did not become generally known until many years later.

One of the very useful notions in many categories as well as in homological algebra is the injectivity of objects with respect to a class $\mathcal{M}$ of morphisms. For example, in the category Pos of partially ordered sets and monotone mappings, injective objects, with respect to the class of all order-embeddings, coincide with the complete lattices (see [4]). Injective objects in slice categories have been investigated in detail (see $[1,16]$ ), especially in relation with weak factorization systems, a concept used in homotopy theory, in particular for model categories.

Received March 7, 2014; Revised September 13, 2014.

2010 Mathematics Subject Classification. Primary 20M30; Secondary 06A11, 18A25, 18G05.

Key words and phrases. S-poset, slice category, regular monomorphism, injectivity. 
More precisely, $\mathcal{M}$-injective objects in $\mathcal{C} / B$ for any $B$ in $\mathcal{C}$, form the right part of a weak factorization system that has morphisms of $\mathcal{M}$ as the left part (see [1]).

In this paper, we investigate $\mathcal{M}$-injective objects in the slice category Pos$S / B$ of $S$-poset maps over $B$, where $\mathcal{M}$ is the class of regular monomorphims in Pos- $S / B$. After some introductory results in Section 1, we give in Section 2 , certain guarantees about the existence of limits and colimits and some categorical properties for the category of Pos- $S / B$. In Section 3, first we show that no non-trivial map is injective as an object in the two categories Pos$S / B$ and Pos $/ B$, but there are enough regular injective objects in Pos- $S / B$ and $\operatorname{Pos} / B$; injective objects with respect to the class $\mathcal{M}$ of regular monomorphisms (morphisms which are equalizers). Also, we show that in the case of Pos- $S / B$, regular monomorphisms correspond to regular monomorphisms in Pos- $S$ and these are exactly order-embeddings (in Pos- $S$ ); that is, $S$-poset maps $f: C \rightarrow D$ for which $f(a) \leq f\left(a^{\prime}\right)$ if and only if $a \leq a^{\prime}$ for all $a, a^{\prime} \in C$. We second find the first relation between $\mathcal{M}$-injective and exponentiable objects in Pos $/ B$ and Pos- $S / B$. In the last section, we restrict ourselves to an object $B$ of the category Pos- $S$ which is endowed with trivial action and supply a partial answer of characterizing regular injectivity in Pos- $S / B$ by observing the relationship with regular injectivity in Pos $/ B$. We prove that, the necessary condition for regular injectivity of an object $f: X \rightarrow B$ in Pos- $S / B$ is the regular injectivity of $f^{-1}(b)$ for every $b \in B$, as objects of Pos- $S$. At the end of the paper, a classification of pogroups, regarding regular injectivity in $\operatorname{Pos}-S / B$, is presented.

In the rest of this section, we give some preliminaries about posets, $S$-acts, $S$-posets and slice categories which we will need in the sequel.

- A poset is said to be complete if each of its subsets has an infimum and a supremum, in particular, a complete poset is bounded, that is, it has the least (bottom) element $\perp$ and the greatest (top) element $T$.

Recall that each poset can be embedded (via an order-embedding) into a complete poset, called the Dedekind-MacNeille completion. In fact, given a poset $P$, its MacNeille completion is the poset $\bar{P}$ consisting of all subsets $A$ of $P$ for which $L U(A)=A$, where $U(A)=\{x \in P: a \leq x, \forall a \in A\}$ and $L U(A)=$ $\{y \in P: y \leq x, \forall x \in U(A)\}$, and the embedding $\varphi: P \rightarrow \bar{P}$ is given by $a \mapsto \downarrow$ $a=\{x \in P: x \leq a\}$ (see [4]).

- Let $S$ be a monoid with identity 1 . Recall that a (right) $S$-act is a set $A$ equipped with a map $\mu: A \times S \rightarrow A$ called its action, such that, denoting $\mu(a, s)$ by $a s$, we have $a 1=a$ and $a(s t)=(a s) t$ for all $a \in A$, and $s, t \in S$. The category of all $S$-acts, with action-preserving ( $S$-act) maps $(f: A \rightarrow B$ with $f(a s)=f(a) s$ for $s \in S, a \in A)$, is denoted by Act- $S$. Clearly $S$ itself is an $S$-act with its operation as the action. For instance, take any monoid $S$ and a non-empty set $A$. Then $A$ becomes a right $S$-act by defining $a s=a$ for all $a \in A, s \in S$, we call that $A$ an $S$-act with trivial action. An element $\theta$ of an $S$-act is called a zero or a fixed element if $\theta s=\theta$ for all $s \in S$. For more information about $S$-acts see [10]. 
- A monoid (semigroup, group) $S$ is said to be a pomonoid (posemigroup, pogroup) if it is also a poset whose partial order $\leq$ is compatible with the binary operation, i.e., $s \leq t, s^{\prime} \leq t^{\prime}$ imply $s s^{\prime} \leq t t^{\prime}$ (see [5]). In this paper $S$ denotes a pomonoid with an arbitrary order, unless otherwise stated.

- Let $S$ be a pomonoid. An (right) $S$-poset is a poset $A$ which is also an $S$-act whose action $\mu: A \times S \rightarrow A$ is order preserving, where we consider $A \times S$ as a poset with componentwise order. The category of all $S$-posets with action preserving monotone maps is denoted by Pos- $S$.

Recall from [6] that in the both categories Pos and Pos- $S$, monomorphisms are exactly one to one morphisms. Also, regular monomorphisms are exactly order-embeddings. We refer the reader to $[6,9]$ for basic results and terminologies in the latter category.

Now, let us recall some categorical notions as we need in sequel.

- Given a category $\mathcal{C}$ and an object $B$ of $\mathcal{C}$, one can construct the slice category $\mathcal{C} / B$ (read: $\mathcal{C}$ over $B$ ): objects of $\mathcal{C} / B$ are morphisms of $\mathcal{C}$ with codomain $B$, and morphisms in $\mathcal{C} / B$ from one such object $f: D \rightarrow B$ to another $g: E \rightarrow B$ are commutative triangles in $\mathcal{C}$

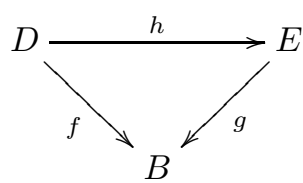

i.e., $g h=f$. The composition in $\mathcal{C} / B$ is defined from the composition in $\mathcal{C}$, in the obvious way. It means paste triangles side by side.

Let $\mathcal{C}$ be a category with finite products. Recall that an object $A$ of $\mathcal{C}$ is called exponentiable if the product functor $-\times A: \mathcal{C} \rightarrow \mathcal{C}$ has a right adjoint (denoted by $(-)^{A}: \mathcal{C} \rightarrow \mathcal{C}$ ). In other words, for every pair of objects $A$ and $B$ of $\mathcal{C}$, an object $B^{A}$ and a morphism ev : $A \times B^{A} \rightarrow B$ exist with the universal property that for every morphism $f: A \times C \rightarrow B$ in $\mathcal{C}$, there exists a unique morphism $\hat{f}: C \rightarrow B^{A}$ such that $\operatorname{ev}\left(\operatorname{id}_{A} \times \hat{f}\right)=f$. In this definition, the object $B^{A}$ is called exponential, ev is said to be the evaluation map, and $\hat{f}$ is called the exponential map associated to $f$. The category $\mathcal{C}$ is called cartesian closed if every object is exponentiable.

Furthermore, for every object $B$ of a category $\mathcal{C}$ with finite products, consider the pullback functor $\prod_{B}: \mathcal{C} \rightarrow \mathcal{C} / B$, which assigns the second projection $\pi_{B}^{X}: X \times B \rightarrow B$ to any object $X$ and the forgetful functor $\sum_{B}: \mathcal{C} / B \rightarrow \mathcal{C}$, which assigns to any $f$ its domain. If $\mathcal{C}$ also has equalizers (i.e., $\mathcal{C}$ has all finite limits), $\prod_{B}$ has a right adjoint if and only if the composition functor $\sum_{B} \circ \prod_{B}$ has a right adjoint. But $\sum_{B} \circ \prod_{B}$ coincides with the product functor $-\times B$, which by definition has a right adjoint when $B$ is exponentiable in $\mathcal{C}$ (see [7]). Thus we have: 
Proposition 1.1. A category $\mathcal{C}$ with finite limits is cartesian closed if and only if for every $B \in \mathcal{C}$, the functor $\prod_{B}: \mathcal{C} \rightarrow \mathcal{C} / B$ has a right adjoint $S_{B}: \mathcal{C} / B \rightarrow \mathcal{C}$.

Following the proof of the previous proposition, for any $f: X \rightarrow B$ with $B$ exponentiable object in a category $\mathcal{C}$ with finite products, the object $S_{B}(f)$ is obtained as the equalizer in $\mathcal{C}$ of the two morphisms $\bar{\gamma}$ and $f^{B}$, where given the morphism $\gamma=\pi_{B}^{X^{B}}: X^{B} \times B \rightarrow B$, by the adjunction we obtain $\bar{\gamma}: X^{B} \rightarrow B^{B}$, which represents a constant morphism of value $\mathrm{id}_{B}$. We might call $S_{B}(f)$ the object of "cross sections of $f$ ". This means that $S_{B}(f)$ can be interpreted as the object of sections of $f$ in $\mathcal{C}$. In every cartesian closed category the authors in [7] present a useful characterization of injective morphisms by the object of sections of these morphisms.

\section{Limits, colimits and some other categorical properties of Pos-S/B}

In this section, we shall characterize limits and colimits in the category Pos$S / B$. We discuss on monomorphisms, epimorphisms and regular monomorphisms. Finally we mention a result to show that the category Pos- $S / B$ is not cartesian closed, in general.

In [6] it has been shown that the category Pos- $S$ has finite limits, so the category Pos- $S / B$ has finite limits too (see [14]), and they are determined as follows. Equalizers in Pos- $S / B$ are formed as in Pos- $S$, that is, the forgetful functor $\sum_{B}$ : Pos- $S / B \rightarrow$ Pos- $S$ which assigns to any $f$ its domain, creates them. We have seen in [6] that the equalizer of a pair $f, g: A \rightarrow B$ of $S$ poset maps is given by $E=\{a \in A: f(a)=g(a)\}$ with action and order inherited from $A$. The terminal object of Pos- $S / B$ is the identity morphism $\operatorname{id}_{B}: B \rightarrow B$. The product of $f: X \rightarrow B$ and $g: Y \rightarrow B$ in Pos- $S / B$ is given by $f \times g: X \times{ }_{B} Y \rightarrow B$, where $X \times{ }_{B} Y$ is the pullback of $f$ and $g$ in Pos- $S$ and $f \times g$ is the obvious projection. Here $X \times_{B} Y$ is the sub-S-poset $\{(x, y) \in X \times Y: f(x)=g(y)\}$ of $X \times Y$.

The category Pos- $S / B$ has finite colimits because Pos- $S$ has (see [6]). In fact, for a category $\mathcal{C}$ with products, a cocone in a category $\mathcal{C} / B$ is a colimit here if and only if the corresponding cocone in $\mathcal{C}$ is a colimit in $\mathcal{C}$. For more information and details see [11].

Remark 2.1. If $f: A \rightarrow C$ is an epimorphism in $\mathcal{C} / B$, then $f$ is an epimorphism in $\mathcal{C}$. Indeed, consider the adjunction $\sum_{B} \dashv \prod_{B}$ where the functors

$$
\sum_{B}: \mathcal{C} / B \rightarrow \mathcal{C} \text { and } \prod_{B}: \mathcal{C} \rightarrow \mathcal{C} / B
$$

defined as in the first section. Since left adjoint functors preserve colimits, the forgetful functor $\sum_{B}$ preserves epimorphisms, so if $f$ is an epimorphism in $\mathcal{C} / B$, then $\sum_{B}(f)=f$ is an epimorphism in $\mathcal{C}$. In the case $\mathcal{C}=$ Pos- $S$, epimorphisms are exactly onto $S$-poset maps (see [6]). Therefore, $f$ is an onto $S$-poset map. 
Remark 2.2. (i) One can prove that every monomorphism in a category $\mathcal{C}$ is exactly a monomorphism in every slice category $\mathcal{C} / B$. Hence monomorphisms in Pos- $S / B$ are one to one $S$-poset maps (see [6]).

(ii) If $f: A \rightarrow C$ is a regular monomorphism in a slice category $\mathcal{C} / B$, then $f$, as a morphism in $\mathcal{C}$, is too. Indeed, $f$ is an equalizer of two arrows $C \rightrightarrows D$ in $\mathcal{C} / B$. On the other hand, equalizer of two arrows in $\mathcal{C} / B$ is just the equalizer in $\mathcal{C}$. Therefore, $f$ is an equalizer of two arrows $C \rightrightarrows D$ in $\mathcal{C}$ too, so $f$ is a regular monomorphism in $\mathcal{C}$.

One might ask whether the converse of (ii) of the above remark holds. However, this is not generally true, but we provide a more practical way to show it for the category Pos- $S$.

Now, we try to prove that regular monomorphisms in Pos- $S / B$ are exactly order-embeddings of the corresponding maps in Pos- $S$. Indeed we have:

Theorem 2.3. Let $S$ be a pomonoid and $B$ an $S$-poset. Moreover, let

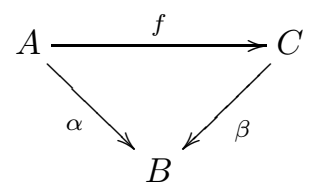

be a morphism in the category Pos-S/B. Then $f$ is a regular monomorphism in Pos- $S / B$ if and only if $f$ is an order-embedding as a map in Pos- $S$.

Proof. The if part follows easily from Remark 2.2(ii) and the fact that regular monomorphisms in Pos- $S$ are exactly order-embeddings (see [6]).

For the only if part, notice that, since $f$ is an order-embedding, the image of $f$ is an $S$-poset which is isomorphic to $A$. So, without loss of generality, we may assume that $f$ is the inclusion map from a sub- $S$-poset $A$ of $C$ into $C$ and prove that $f$ is a regular monomorphism. As we remarked at the beginning of this section, a pushout of two morphisms in Pos- $S / B$ is just the pushout in Pos-S. So take the amalgamated coproduct $C \coprod_{A} C$ (i.e., pushout $f$ with itself) and the obvious morphisms $g_{1}, g_{2}: C \rightarrow C \coprod_{A} C$. Then $f$ is clearly the equalizer of $g_{1}$ and $g_{2}$. Now by pushout property there exists a unique $S$-poset map $h: C \coprod_{A} C \rightarrow B$ such that $h g_{1}=h g_{2}=\beta$. Then it is easily seen that $f$ is the equalizer of $g_{1}, g_{2}$ in Pos- $S / B$.

We close this section by checking cartesian closedness of the category Pos$S / B$.

The category Pos- $S / B$ is not necessarily cartesian closed. Indeed, let $S=$ $\{1\}$ be the trivial monoid. Then the category Pos- $S / B$ is isomorphic to the category Pos $/ B$ and this category is not cartesian closed (see [15]).

\section{Injectivity and regular injectivity}

Let $\mathcal{C}$ be a category and $\mathcal{M}$ a class of its morphisms. An object $I$ of $\mathcal{C}$ is called $\mathcal{M}$-injective if for each $\mathcal{M}$-morphism $h: U \rightarrow V$ and morphism $u: U \rightarrow I$ 
there exists a morphism $s: V \rightarrow I$ such that $s h=u$. That is, the following diagram is commutative:

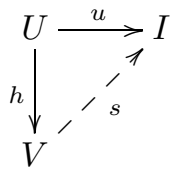

In particular, this means that, in the slice category $\mathcal{C} / B, f: X \rightarrow B$ is $\mathcal{M}$ injective if, for any commutative diagram

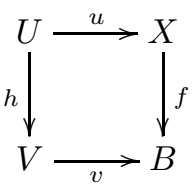

with $h \in \mathcal{M}$, there exists an arrow $s: V \rightarrow X$ such that $s h=u$ and $f s=v$ :

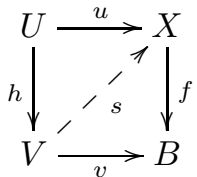

It is clear that the terminal object (if exist) is $\mathcal{M}$-injective in any category. It is called the trivial injective object.

There are two main questions about notion of $\mathcal{M}$-injectivity in a category $\mathcal{C}:$

(1) Does $\mathcal{C}$ have enough $\mathcal{M}$-injective objects? That is for any $A \in \mathcal{C}$, does there exist an $\mathcal{M}$-injective $I \in \mathcal{C}$ with an $\mathcal{M}$-morphism $A \rightarrow I$ ?

(2) Is it true that $I$ is $\mathcal{M}$-injective if and only if it is absolutely $\mathcal{M}$-retract (i.e., any $\mathcal{M}$-morphism $I \rightarrow A$ has a left inverse in $\mathcal{C}$ )?

We now study injectivity and regular injectivity in the category Pos- $S / B$. We start with the following example.

Example 3.1. Consider the category Pos- $S / B$ and let $\mathcal{U}$ be the class of all unitary $S$-poset monomorphisms (recall [2] that an $S$-poset monomorphism $f: X \rightarrow Y$ is unitary if $y \in \operatorname{im}(f)$, whenever $y s \in \operatorname{im}(f)$ for some $s \in S)$ and $\mathcal{E}_{S}$ be the class of all split $S$-poset epimorphisms (that is, an epimorphism that has a right inverse). One can prove that, each $g \in \mathcal{E}_{S}$ is $\mathcal{U}$-injective in this category. Also, we can show that $\left(\mathcal{U}, \mathcal{E}_{S}\right)$ is a weak factorization system for the category Pos- $S$. Details of proof is similar to Theorem 3.1 in [2].

We state two results from [3] that will be used in the following.

Lemma 3.2. Let $F: \mathcal{C} \rightarrow \mathcal{D}$ and $G: \mathcal{D} \rightarrow \mathcal{C}$ be two functors such that $F \dashv G$. Also, let $\mathcal{M}$ and $\mathcal{N}$ be certain subclasses of $\mathcal{C}$ and $\mathcal{D}$, respectively. If for all $f \in \mathcal{M}, F f \in \mathcal{N}$, then for any $\mathcal{N}$-injective object $D$ of $\mathcal{D}, G D$ is an $\mathcal{M}$-injective object of $\mathcal{C}$. 
Corollary 3.3. If, in addition, $F$ is faithful and $\mathcal{D}$ has enough injective objects, then $\mathcal{C}$ has enough injective objects.

Now, we have:

Lemma 3.4. If $I$ is $\mathcal{M}$-injective in a category $\mathcal{C}$ with finite products, then the second projection $\pi_{B}^{I}: I \times B \rightarrow B$ is $\mathcal{M}$-injective in the slice category $\mathcal{C} / B$ for every $B \in \mathcal{C}$.

Proof. It is clear that the forgetful functor $\sum_{B}: \mathcal{C} / B \rightarrow \mathcal{C}$ preserves $\mathcal{M}$ morphisms. Now, by adjunction $\sum_{B} \dashv \prod_{B}$ and Lemma 3.2, the proof is clear.

Corollary 3.5. Let $\mathcal{C}$ be a cartesian closed category. Then $S_{B}: \mathcal{C} / B \rightarrow \mathcal{C}$, the right adjoint of the pullback functor $\prod_{B}: \mathcal{C} \rightarrow \mathcal{C} / B$, preserves injectivity and regular injectivity.

Proof. As we have mentioned, the pullback functor $\prod_{B}: \mathcal{C} \rightarrow \mathcal{C} / B$ has a left adjoint $\sum_{B}$, so it preserves all limits especially monomorphisms and regular monomorphisms. On the other hand, since the category $\mathcal{C}$ is cartesian closed, we can apply the adjunction $\prod_{B} \dashv S_{B}$ as in Proposition 1.1. Therefore, in view of Lemma 3.2, the functor $S_{B}$ preserves injectivity and regular injectivity, as required.

Recall from [6] that Pos- $S$ (especially Pos) is cartesian closed. As the two categories Pos and Pos- $S$ have no non-trivial injective objects (see [8]), so by Corollary 3.5 we immediately obtain the following result:

Theorem 3.6. The categories Pos $/ B$ and Pos-S/B have no non-trivial injective object.

\subsection{Regular injectivity and completeness}

Let $\mathcal{C}$ be a category. From now on, 'regular injective' will mean $\mathcal{M}$-injective for $\mathcal{M}$ the class of regular monomorphisms in $\mathcal{C}$.

It is well known that both categories Pos and Pos- $S$ have enough regular injective objects (see $[4,8]$ ). In the following, we give a positive answer to the first question above regarding the two categories Pos $/ B$ and Pos- $S / B$.

Definition 3.7. By a complete $S$-poset, we mean an $S$-poset which is merely complete as a poset.

Theorem 3.8. For an arbitrary $S$-poset $B$, the category Pos-S/B has enough regular injective objects. More precisely, each object $f: A \rightarrow B$ in Pos-S/B can be regularly embedded to a regular injective object $\pi_{B}^{\bar{A}^{(S)}}: \bar{A}^{(S)} \times B \rightarrow B$ in Pos$S / B$ in which $\bar{A}^{(S)}$ is the set of all monotone maps from $S$ to $\bar{A}$, with pointwise order and the action given by $(f s)(t)=f(s t)$ for $s, t \in S$ and $f \in \bar{A}^{(S)}$ and $\pi_{B}^{\bar{A}^{(S)}}: \bar{A}^{(S)} \times B \rightarrow B$ is the second projection. 
Proof. Let $f: A \rightarrow B$ be an object of Pos- $S / B$. Then we consider the Dedekind-MacNeille completion $\bar{A}$ of $A$. It was proved in [8] that $\bar{A}^{(S)}$ is a regular injective $S$-poset. From Lemma 3.4 , the second projection $\pi_{B}^{\bar{A}^{(S)}}$ : $\bar{A}^{(S)} \times B \rightarrow B$ is a regular injective object in the category Pos- $S / B$. Now, the mapping $\langle\varphi, f\rangle: A \rightarrow \bar{A}^{(S)} \times B$ given by $\langle\varphi, f\rangle(a)=\left\langle\varphi_{a}, f(a)\right\rangle$ for every $a \in A$, where $\varphi_{a}: S \rightarrow \bar{A}$ defined by $\varphi_{a}(s)=\downarrow(a s)$, is the required orderedembedding $S$-poset map (that is, a regular monomorphism in Pos- $S / B$ ) such that $\pi_{B}^{\bar{A}^{(S)}}\langle\varphi, f\rangle=f$. The proof now is complete.

Similar to Theorem 3.8 we have the following result:

Theorem 3.9. For an arbitrary poset B, the category Pos/B has enough regular injective objects. More precisely, each object $f: A \rightarrow B$ in Pos $/ B$ can be regularly embedded into a regular injective object $\pi_{B}^{\bar{A}}: \bar{A} \times B \rightarrow B$ in Pos $/ B$ in which $\bar{A}$ is the Dedekind-MacNeille completion of $A$ and $\pi_{B}^{\bar{A}}$ is the second projection.

Proof. We take $S=\{1\}$ in Theorem 3.8, then we get our result for the category $\operatorname{Pos} / B$.

In [8], it has been shown that, if $S$ is a pogroup, then regular injective object and complete $S$-poset in Pos- $S$ are the same. By this fact and Lemma 3.4, we deduce:

Proposition 3.10. Let $S$ be a pogroup and $A$ a complete $S$-poset. Then the second projection $\pi_{B}^{A}: A \times B \rightarrow B$ is a regular injective object in Pos-S/B.

Corollary 3.11. Let $S$ be a pogroup and $A$ a complete $S$-poset. Then each $f: A \rightarrow B$ in Pos- $S / B$ can be regularly embedded into $\pi_{B}^{A}: A \times B \rightarrow B$, which is a regular injective object in Pos $-S / B$.

Proof. It is easily seen that the map $g: A \rightarrow A \times B$, given by $g(a)=\langle a, f(a)\rangle$, for every $a \in A$, is the required order-embedding $S$-poset map with $\pi_{B}^{A} g=$ $f$.

\subsection{Regular injectivity and exponentiability}

Let us first recall the following proposition which comes from [13]. It will be used in our next results.

Proposition 3.12. Let $A$ be an exponentiable object in a category $\mathcal{C}$ with finite products. Then $\prod_{B}(A)$ is exponentiable in the slice category $\mathcal{C} / B$ for every $B \in \mathcal{C}$.

As a consequence, we find the first relationship between injective and exponentiable objects in Pos $/ B$ and Pos- $S / B$ :

Theorem 3.13. In the category Pos-S/B, any regular injective object is a retract of an exponentiable object. 
Proof. Let $f: A \rightarrow B$ be a regular injective object in Pos- $S / B$. By Theorem 3.8 , we get the following commutative diagram:

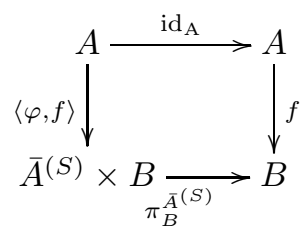

By the assumption, there exists an arrow $s: \bar{A}^{(S)} \times B \rightarrow A$ such that $s\langle\varphi, f\rangle=$ $\operatorname{id}_{\mathrm{A}}$ and $f s=\pi_{B}^{\bar{A}^{(S)}}$ :

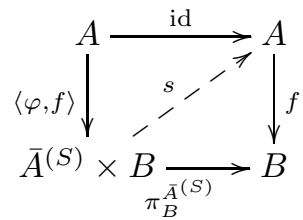

Here, $\langle\varphi, f\rangle: f \rightarrow \pi_{B}^{\bar{A}^{(S)}}$ and $s: \pi_{B}^{\bar{A}^{(S)}} \rightarrow f$ are two morphisms in Pos- $S / B$ such that $s\langle\varphi, f\rangle=\operatorname{id}_{f}$.

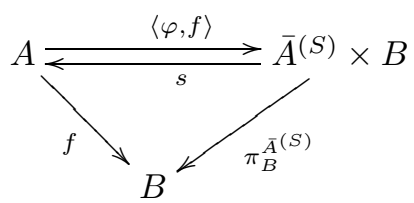

Hence $f$ is a retract of $\pi_{B}^{\bar{A}^{(S)}}$ in Pos-S/B, whereas $\pi_{B}^{\bar{A}^{(S)}}$ is an exponentiable object in Pos- $S / B$. In fact, because Pos- $S$ is a cartesian closed category, $\bar{A}^{(S)}$ is an exponentiable object in Pos- $S$ and by Proposition 3.12, $\pi_{B}^{\bar{A}^{(S)}}=\Pi_{B}\left(\bar{A}^{(S)}\right)$ is an exponentiable object in Pos- $S / B$. This completes the proof.

We remark that if we take $S=\{1\}$, then in view of Theorem 3.9, we deduce that the above theorem is true for the category Pos $/ B$.

Notice that for every category with enough $\mathcal{M}$-injectivity property the answer to the second question at the beginning of this section is always true. However, we review its proof for the slice category Pos- $S / B$.

Theorem 3.14. Let $B$ be an arbitrary $S$-poset and $f: A \rightarrow B$ be an object in Pos-S/B. Then $f$ is regular injective object if and only if every regular monomorphism $m$

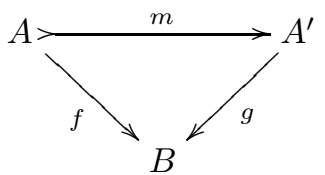

in Pos-S/B has a left inverse (i.e., $f$ is absolute retract in $\mathbf{P o s}-S / B$ ). 
Proof. For necessary part, consider the following commutative diagram:

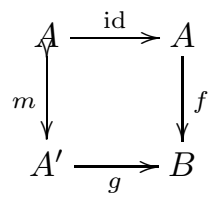

By regular injectivity of $f$, there exists an arrow $s: A^{\prime} \rightarrow A$, such that $f s=g$ and $s m=\mathrm{id}_{A}$. Therefore $m$ has a left inverse $s$. For sufficiency part, take a regular monomorphism $h: C \rightarrow C^{\prime}$ satisfying in the following commutative diagram:

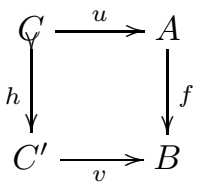

By Theorem 3.8, $f$ can be regularly embedded into the regular injective object $\pi_{B}^{\bar{A}^{(S)}}: \bar{A}^{(S)} \times B \rightarrow B$ via $\langle\varphi, f\rangle$. Since $\pi_{B}^{\bar{A}^{(S)}}$ is regular injective in the category Pos- $S / B$, so by the following commutative diagram

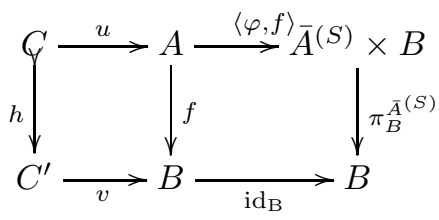

there exists an arrow $\psi: C^{\prime} \rightarrow \bar{A}^{(S)} \times B$ such that $\pi_{B}^{\bar{A}^{(S)}} \psi=\mathrm{id}_{B} v$ and $\psi h=$ $\langle\varphi, f\rangle u$. Also, by hypothesis, there exists a retraction $k: \bar{A}^{(S)} \times B \rightarrow A$ such that $f k=\pi_{B}^{\bar{A}^{(S)}}$. Now, $g:=k \psi$ is an $S$-poset map with $f g=\pi_{B}^{\bar{A}^{(S)}} \psi=v$ and $g h=k \psi h=k\langle\varphi, f\rangle u=u$.

\subsection{Injectivity in Pos-S/B in which $B$ is endowed with trivial action}

In this subsection, we are going to find characterization of injective morphisms in the category Pos- $S / B$. In this part, we supply a partial answer to the characterization of regular injectivity in the category Pos- $S / B$ in a special case, when the $S$-poset $B$ has trivial action.

We state the following theorem from [7] which is true, of course, for every cartesian closed category.

Theorem 3.15. Let $\prod_{B} \dashv S_{B}: \mathcal{C} / B \rightarrow \mathcal{C}$. Then $f: X \rightarrow B$ is a regular injective object in $\mathcal{C} / B$ if and only if the following two conditions are satisfied:

(1) $\left\langle 1_{X}, f\right\rangle: f \rightarrow \pi_{B}^{X}$ is a section in $\mathcal{C} / B$.

(2) The object $S_{B}(f)$ of sections of $f$ is a regular injective object in $\mathcal{C}$.

Remark 3.16. For a pomonoid $S$, we know that Pos- $S$ is cartesian closed (see [6]). Indeed, given two $S$-posets $A$ and $B$, the exponential $B^{A}$ is given by 
$B^{A}=\operatorname{hom}(S \times A, B)$, the set of all $S$-poset maps from the product $S$-poset $S \times A$ to $B$. Note that the action on $S \times A$ operates on both components. This set is an $S$-poset, with pointwise order and the action is given by $(f s)(t, a)=f(s t, a)$ (see $[6,12]$ ). Now, given $f: X \rightarrow B$ in Pos- $S / B$ we have

$$
S_{B}(f)=\left\{h \in \operatorname{hom}(S \times B, X) \mid f h=\pi_{B}^{S}\right\} .
$$

Proposition 3.17. Let $S$ be a pomonoid. If $f: X \rightarrow B$ is a regular injective object in the category Pos-S/B in which $B$ has trivial action, then:

(i) $\left\langle 1_{X}, f\right\rangle$ is a section in Pos-S/B.

(ii) for every $b \in B$, the sub $S$-poset $f^{-1}(b)$ of $X$ is a regular injective object in Pos-S, so it is a complete poset.

Proof. Let $f: X \rightarrow B$ be a regular injective object in the category Pos- $S / B$ in which $B$ has trivial action. Since Pos- $S$ is a cartesian closed category, by Theorem 3.15 condition (1) holds. Let $b \in B$. As $B$ had trivial action it is easy to see that $f^{-1}(b)=\{x \in X \mid f(x)=b\}$ is a sub $S$-poset of $X$. We must show that $f^{-1}(b)$ is a regular injective object in Pos- $S$. Given the following diagram<smiles>CC=C[Tl]</smiles>

where $g$ is a regular monomorphism in Pos- $S$. We complete it to the following commutative diagram

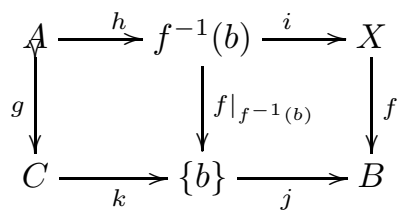

in which $i$ and $j$ are inclusion maps and $k$ is the constant map with value $b$. Note that $k: C \rightarrow\{b\}$ is an $S$-poset map, because the action of $B$ is trivial. Now, by assumption, there exists an $S$-poset map $\varphi: C \rightarrow X$ such that $f \varphi=j k$ and $\varphi g=i h$. From $f \varphi=j k$, one can show that $\operatorname{im} \varphi \subseteq f^{-1}(b)$. Also, from $\varphi g=i h$ we get the following commutative diagram:

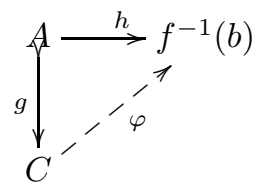

We conclude that $f^{-1}(b)$ is a regular injective object in Pos- $S$.

Corollary 3.18. Let $S$ be a pomonoid and $f: X \rightarrow B$ an object in Pos-S/B. If $f$ is a regular injective object in $\mathbf{P o s}-S / B$, then it is a regular injective object in Pos/B. 
Proof. First of all, by hypothesis and statement (ii) of Proposition 3.17, each $f^{-1}(b)$ is a complete poset, where $b \in B$. Therefore it is a regular injective object in Pos by [4]. Next, in view of Theorem 2.1 of [7] and statement (i) of Proposition 3.17, we deduce that $f$ is a regular injective object in Pos $/ B$.

For the next result first we need a definition which we mention it from [1].

Definition 3.19. A map $f: X \longrightarrow B$ between posets is said to be convex if for any $x$ preceding $y$ in $X$, every $b$ between $f(x)$ and $f(y)$ in $B$ can be lifted to an element between $x$ and $y$ in $X$.

Proposition 3.20. Let $S$ be a pomonoid. If $f: X \rightarrow B$ is a regular injective object in Pos- $S / B$, then $f$ is convex as a map in Pos- $S$.

Proof. By the previous corollary, $f$ is a regular injective object in Pos/B. Then it has been shown in [1] that such $f$ must be convex.

Next, we present an example to show that the converse of the previous result is not true, in general.

Example 3.21. Let $S$ be an arbitrary pomonoid and $X, B$ be two posets as shown in the following from left to right, respectively.
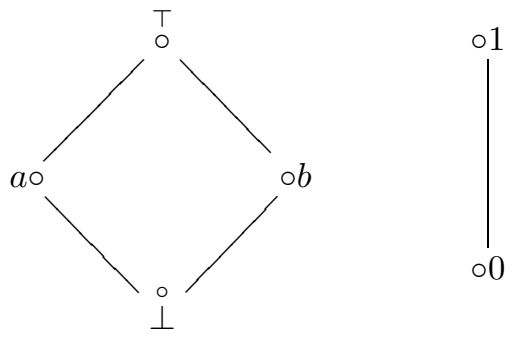

Then $X$ is an $S$-poset with the action defined by $\top s=\top$ and $a s=b s=\perp s=a$ for all $s \in S$, also we consider $B$ with the trivial action as an $S$-poset. Define the $S$-poset map $f: X \rightarrow B$ in Pos- $S / B$, by $f(a)=f(b)=f(\perp)=0$ and $f(\top)=1$. It also is a convex map. Since $f^{-1}(0)=\{\perp, a, b\}$ is not a complete poset, Proposition 3.17 shows that it is not regular injective object in Pos- $S / B$.

To prove the next result we need the following lemma from [8].

Lemma 3.22. Let $S$ be a pogroup and $A$ be a complete $S$-poset. Then, for any $X \subseteq A$ and $s \in S,(\bigvee X) s=\bigvee\{x s \mid x \in X\}$.

Theorem 3.23. Let $S$ be a pogroup. An object $f: X \rightarrow B$ in Pos-S/B is a regular injective object if and only if the following two conditions are satisfied:

(1) $\left\langle 1_{X}, f\right\rangle$ is a section in Pos-S/B.

(2) The sub $S$-posets $f^{-1}(b)$ of $X$, in which $b \in B$, are regular injective objects in Pos-S, that is, every $f^{-1}(b)$ is a complete poset. 
Proof. The necessity condition follows from the previous proposition. For sufficiency, suppose that both conditions (1) and (2) are hold. Then, in view of Theorem 3.15, and Theorem 4.2 of [8], it is enough to show that $S_{B}(f)$ is a complete poset. In fact, since by assumption $S$ is a pogroup, it is a regular injective object in the category Pos- $S$ (see [8]). Let $\left\{g_{i}: i \in I\right\}$ be an arbitrary subset of $S_{B}(f)$ (see Remark 3.16). Then for any $i \in I$ and for any $(s, b) \in S \times B, g_{i}(s, b) \in f^{-1}(b)$. On the other hand, any $f^{-1}(b)$ is a complete poset and hence we can take $\bigvee_{f^{-1}(b)}\left\{g_{i}(s, b) \mid i \in I\right\}$. Next, we define a map $g: S \times B \rightarrow X$ by the rule

$$
g(s, b):=\bigvee_{f^{-1}(b)}\left\{g_{i}(s, b) \mid i \in I\right\} .
$$

This map is order preserving. In fact, if $\left(s_{1}, b_{1}\right) \leq\left(s_{2}, b_{2}\right)$, then $g_{i}\left(s_{1}, b_{1}\right) \leq$ $g_{i}\left(s_{2}, b_{2}\right)$ for every $i \in I$. Since $g_{i}\left(s_{2}, b_{2}\right) \in f^{-1}\left(b_{2}\right)$, so

$$
g_{i}\left(s_{1}, b_{1}\right) \leq g_{i}\left(s_{2}, b_{2}\right) \leq \bigvee_{f^{-1}\left(b_{2}\right)}\left\{g_{i}\left(s_{2}, b_{2}\right) \mid i \in I\right\}
$$

for every $i \in I$. Again, since $g_{i}\left(s_{1}, b_{1}\right) \in f^{-1}\left(b_{1}\right)$ for every $i \in I$, so

$$
\bigvee_{f^{-1}\left(b_{1}\right)}\left\{g_{i}\left(s_{1}, b_{1}\right) \mid i \in I\right\} \leq \bigvee_{f^{-1}\left(b_{2}\right)}\left\{g_{i}\left(s_{2}, b_{2}\right) \mid i \in I\right\}
$$

Therefore, $g\left(s_{1}, b_{1}\right) \leq g\left(s_{2}, b_{2}\right)$.

Also, this map is action-preserving. Because, for every $(s, b) \in S \times B, t \in S$, we have

$$
\begin{aligned}
(g(s, b)) t & =\left(\bigvee_{f^{-1}(b)}\left\{g_{i}(s, b) \mid i \in I\right\}\right) t \\
& =\bigvee_{f^{-1}(b)}\left\{\left(g_{i}(s, b) t\right) \mid i \in I\right\} \\
& =\bigvee_{f^{-1}(b)}\left\{g_{i}(s t, b t) \mid i \in I\right\} \\
& =\bigvee_{f^{-1}(b)}\left\{g_{i}(s t, b) \mid i \in I\right\} \\
& =g(s t, b) \\
& =g(s t, b t) \\
& =g((s, b) t),
\end{aligned}
$$

where the second equality comes from Lemma 3.22 and completeness of $f^{-1}(b)$ as an $S$-poset. Moreover, this $S$-poset map is in $S_{B}(f)$, since for every $b \in B$, $f^{-1}(b)$ is a complete poset, so $f g(s, b)=f(g(s, b))=b=\pi_{B}^{S}(s, b)$. Therefore $f g=\pi_{B}^{S}$. On the other hand, Pos- $S$ is cartesian closed hence Proposition 1.1 and Theorem 3.15 allow us to say that $f$ is regular injective in Pos- $S / B$.

Acknowledgments. The authors would like to thank the anonymous referees for their careful reading of the manuscript and useful comments. Also, we acknowledge the kind hospitality of Hakim Sabzevari University, during our stay there, which helped us to finish this paper. 


\section{References}

[1] J. Adamek, H. Herrlich, J. Rosicky, and W. Tholen, Weak factorization systems and topological functors, Appl. Categ. Structures 10 (2002), no. 1, 237-249.

[2] A. Bailey and J. Renshaw, Weak factorization system for $S$-acts, Semigroup Forum 89 (2014), no. 1, 52-67.

[3] B. Banaschewski, Injectivity and essential extensions in equational classes of algebras, Proc. Conf. on Universal Algebra (Queen's Univ., Kingston, Ont., 1969) pp. 131-147 Queen's Univ., Kingston, Ont. 1970.

[4] B. Banaschewski and G. Bruns, Categorical characterization of the MacNeille completion, Arch. Math. 18 (1967), 369-377.

[5] G. Birkhoff, Lattice Theory, American Mathematical Society, Providence, 1973.

6] S. Bulman-Fleming and M. Mahmoudi, The category of S-posets, Semigroup Forum 71 (2005), no. 3, 443-461.

[7] F. Cagliari and S. Mantovani, Injectivity and sections, J. Pure Appl. Algebra 204 (2006), no. $1,79-89$.

[8] M. M. Ebrahimi, M. Mahmoudi, and H. Rasouli, Banaschewski's theorem for S-posets: regular injectivity and completeness, Semigroup Forum 80 (2010), no. 2, 313-324.

[9] S. M. Fakhruddin, On the category of S-posets, Acta Sci. Math. (Szeged) 52 (1988), no. 1-2, 85-92.

[10] M. Kilp, U. Knauer, and A. Mikhalev, Monoids, Acts and Categories, de Gruyter, Berlin, 2000.

[11] S. Mac Lane and I. Moerdijk, Sheaves in Geometry and Logic: A First Introduction to Topos Theory, Springer Universitext, New York, 1992.

[12] A. Madanshekaf and J. Tavakoli, Tiny objects in the category of $M$-Sets, Ital. J. Pure Appl. Math. 10 (2001), 153-162.

[13] C. McLarty, Elementary Categories, Elementary Toposes, Oxford University Press, New York, 1992 .

[14] S. Niefield, Cartesianness: topological spaces, uniform spaces and affine schemes, J. Pure Appl. Algebra 23 (1982), no. 2, 147-167.

[15] _ Exponentiable morphisms: posets, spaces, locals, and Grothendieck toposes, Theory Appl. Categ. 8 (2001), 16-32.

[16] W. Tholen, Injectives, exponentials, and model categories, In: Abstracts of the Int. Conf. on Category Theory, 183-190, Como, Italy, 2000.

FARIDEH FARSAD

Department of Mathematics

SEMNAN UNIVERSITY

P. O. Box 35131-19111, Semnan, Iran

E-mail address: faridehfarsad@yahoo.com

Ali MadansheKaF

Department of Mathematics

SEmnan University

P. O. Box 35131-19111, SEMnAN, IrAN

E-mail address: amadanshekaf@semnan.ac.ir 